Pirineos, 163: 77 a 84, JACA; 2008. ISSN 0373-2568

\title{
CONSECUENCIAS DEL RETROCESO GLACIAR EN LA CORDILLERA BOLIVIANA
}

\author{
D. HOFFMANN \\ Instituto Boliviano de la Montaña. Casilla 3-12417. La Paz, Bolivia. Telf. y Fax: +591-2-2712432. \\ c.e.: dirk.hoffmann@bolivian-mountains.org
}

\begin{abstract}
Glaciers have proven to be an excellent indicator of climate variations throughout the Holocene there are a number of studies on the recent trend towards disappearance of Bolivian glaciers. But at present, practically no research has been carried out on the consequences the melting and eventual disappearance of the country's glaciers has and might have on the economic and social situation of its population. Thus, this country study on socio-economic impacts of glacier retreat is a first approach to get an overview of what and where might be expected in the future. Bolivian glaciers represent around $20 \%$ of the world's tropical glacier area (Jordan 1991). The increased melting and disappearance started at the beginning of the 80s, with another increase during the last 5-10 years. Thus it is probable that most of the smaller glaciers which have not already vanished will have completely disappeared within 10 to 20 years from today. Impacts of glacier retreat are most likely to be mainly local, in some cases regional, which means that local authorities must play a vital role in this context. The supply of (drinking) water for the growing urban conglomeration La Paz - El Alto, is the main issue with shrinking glaciers in Bolivia.
\end{abstract}

Key words: Glacial retreat, hydric resources, socio-economic impacts, Bolivia.

RESUMEN.- A la fecha existe un importante número de estudios que demuestran la tendencia actual hacia la desaparición de los glaciares bolivianos. El presente estudio del impacto socio-económico del retroceso de los glaciares es un primer intento de obtener un panorama sobre dónde y qué cambios se podría esperar en un futuro cercano. Aproximadamente el $80 \%$ de los glaciares bolivianos tienen una superficie menor a 0,5 $\mathrm{km}^{2}$; por lo tanto, es muy probable que la mayoría de ellos hayan desaparecido completamente durante los próximos 10 a 15 años. Los impactos socio-económicos se harán notar sobre todo en un nivel local, y los elementos más preocupantes son, por un lado, la falta de investigación sobre el tema y por otro, la falta de una conciencia pública sobre el problema en la población boliviana y que una parte socio-políticamente importante 
y numerosa de la población será probablemente más afectada, la metrópoli La Paz - El Alto.

Palabras clave: Retroceso glaciar, recursos hidráulicos, impactos socioeconómicos, Bolivia.

\section{Introducción: Los glaciares bolivianos}

Los glaciares han demostrado ser un excelente indicador de las variaciones climáticas durante el Holoceno. A la fecha, un importante número de estudios, que analizando las múltiples interacciones de las condiciones geo-meteorológicas de las altas montañas del sub-trópico boliviano, demuestran la tendencia actual hacia la desaparición de los glaciares (RAMÍREZ et al., 2001); sin embargo, hasta el momento no se ha llevado a cabo ninguna investigación sobre las consecuencias que el deshielo y posible desaparición de los glaciares bolivianos podría tener sobre la situación económica y social de la población.

En países en vías de desarrollo, hay poca capacidad instalada para el desarrollo de estudios no relacionados directamente a problemas del desarrollo o del bienestar de la gente. De esta forma el estudio del impacto socio-económico del retroceso de los glaciares conducido por HOFFMANN (2005) significa un primer intento de obtener un panorama general sobre los lugares y los cambios que se podría esperar en un futuro, tomando como base la velocidad actual del deshielo de los pequeños glaciares de los Andes bolivianos.

Según los datos del World Glacier Monitoring Service, en América del Sur existen $25.908 \mathrm{~km}^{2}$ del total mundial de $15.861 .766 \mathrm{~km}^{2}$ de superficie glaciar (WGMS, 2007), que representa menos del 0,2\%. De este monto, unos $566 \mathrm{~km}^{2}$ pertenecen a los Andes bolivianos, que representa poco más del $2 \%$ de toda la superficie glaciar de América Latina.

Desde otra perspectiva vemos que, los glaciares bolivianos representan aproximadamente el $20 \%$ de todos los glaciares tropicales del mundo (JORDAN, 1991).

El área máxima alcanzado por los glaciares bolivianos durante la Pequeña Época de Hielo (LIA-Little Ice Age), era un 50\% más grande que a la fecha (datos de 1975), (JORDAN, 1991).

El incremento acelerado del deshielo de los glaciares comenzó a inicios de los años 80, con otro incremento durante los últimos 5-10 años (Figura 1). Al igual que en otras regiones, los pequeños glaciares son más sensibles hacia el cambio climático, y están desapareciendo a una mayor velocidad. Como el 
$80 \%$ aproximadamente de los 1.830 glaciares bolivianos tienen una superficie menor a $0,5 \mathrm{~km}^{2}$ (JORDAN, 1991), es muy probable que la mayoría de ellos hayan desaparecido completamente durante los próximos 10 a 15 años.

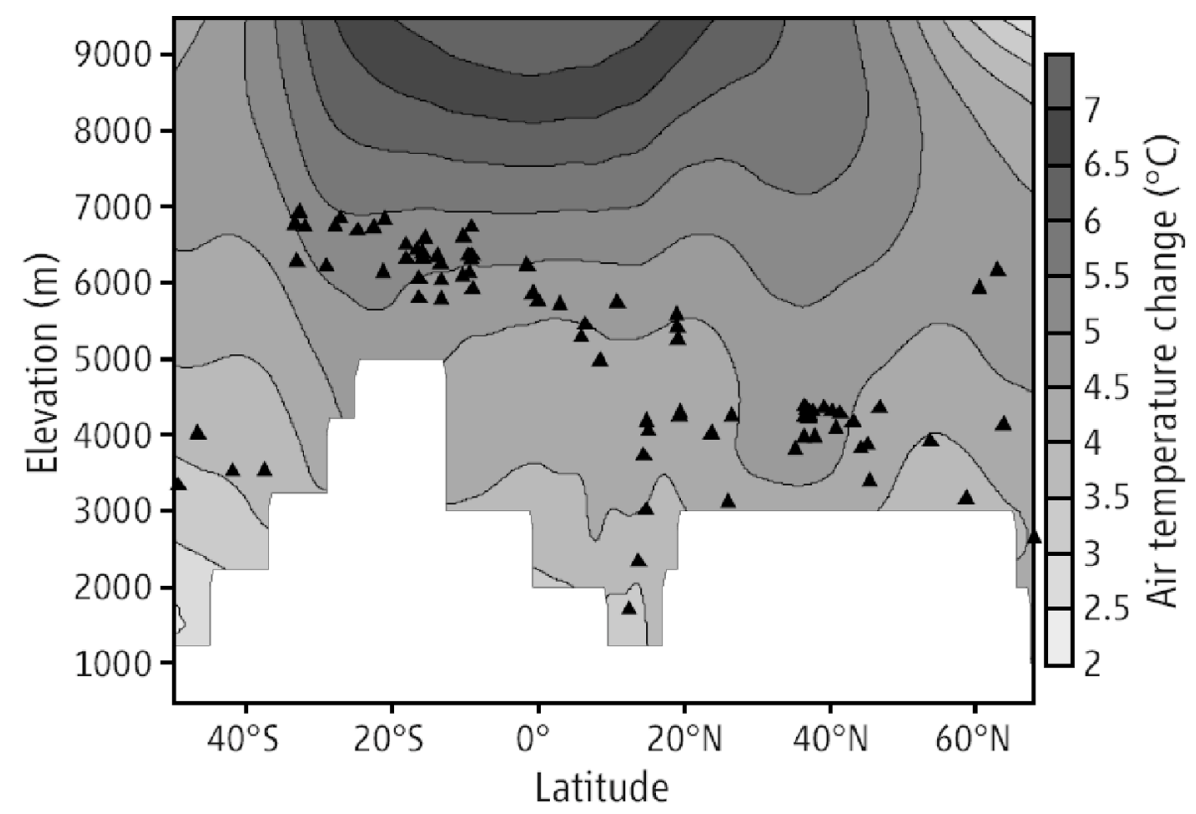

Figura 1. Calentamiento global en la Cordillera Americana. BRADLEY et al., (2006).

\section{2. Área de estudio}

El área de estudio abarca todas las regiones de cordillera donde existen glaciares. Casi la totalidad de los glaciares bolivianos se encuentran en la Cordillera Oriental (de norte a sur: Cordillera de Apolobamba, Cordillera Real, Cordillera Quimsa Cruz, Cordillera Santa Vera Cruz), con solo unos pequeños glaciares en la Cordillera Occidental (Nevado Sajama, Nevados Payachatas) (Tabla 1 y Fig. 2).

\section{Aspectos metodológicos}

Hasta el presente no se ha encontrado ninguna evidencia, escrita $u$ oral, que documente o testimonie que el derretimiento de los glaciares haya 
D. HOFFMANN

Tabla 1. Distribución de los glaciares por las cordilleras bolivianas. Elaboración propia en base a los datos de Jordan (1991).

\begin{tabular}{|l|c|}
\hline Cordillera & Porcentaje \\
\hline Cordillera Occidental & $2 \%$ \\
\hline Cordillera Oriental & \\
\hline Cordillera Apolobamba & $37 \%$ \\
\hline Cordillera Real & $55 \%$ \\
\hline $\begin{array}{l}\text { Cordillera Quimsa Cruz } \\
\text { (incluye Santa Vera Cruz) }\end{array}$ & $8 \%$ \\
\hline
\end{tabular}

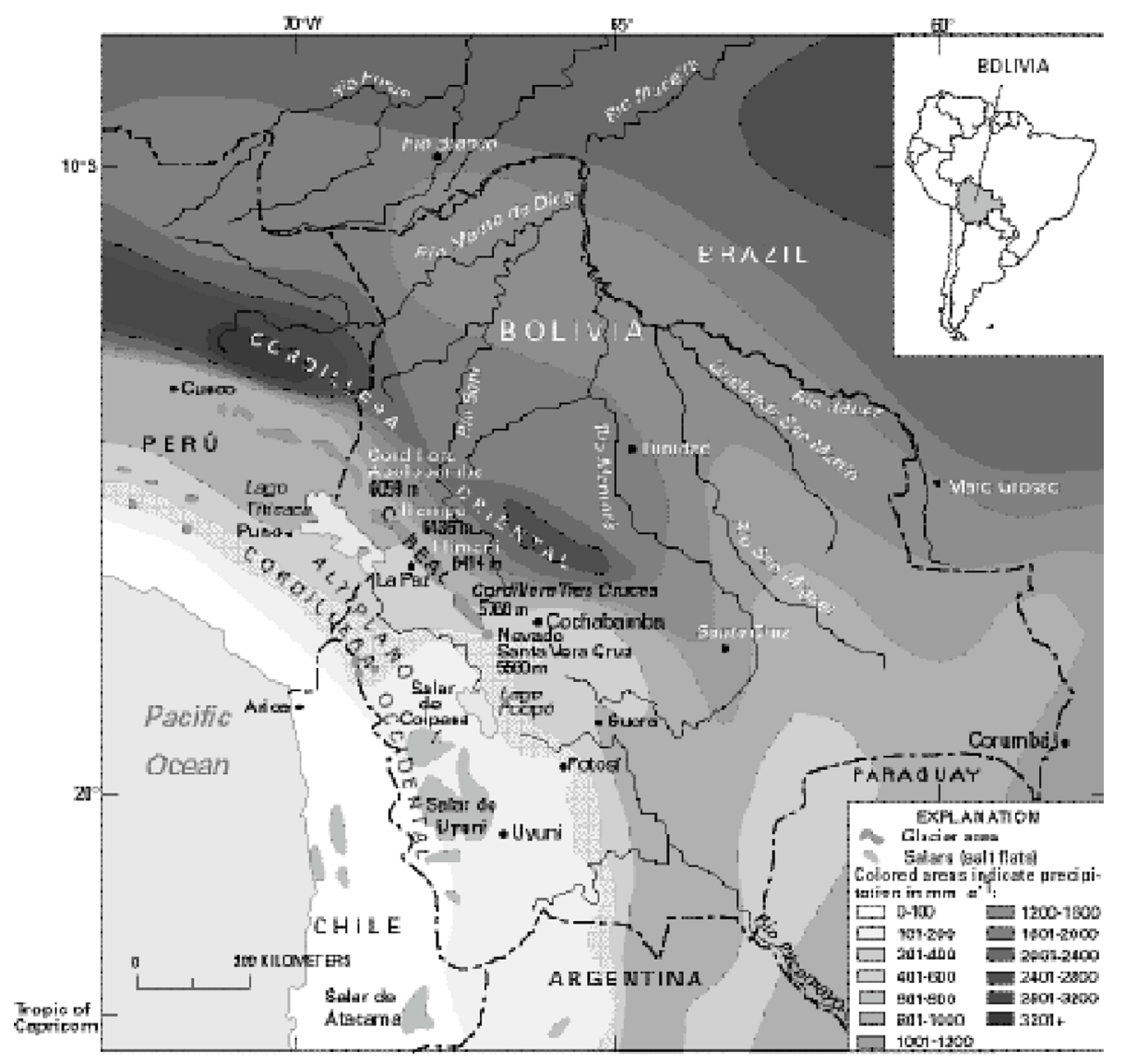

Figura 2. Ubicación de los glaciares en Bolivia. Fuente: WGMS 2007. 
producido algún impacto significativo sobre actividades humanas. Además, la falta de datos confiables u otros investigaciones acerca de la temática en el país, ha hecho necesario analizar la evidencia de impactos socio-económicos de otros países y regiones para poder identificar varios tipos o categorías de los posibles impactos.

En base de una matriz, se han enlistado todos los posibles impactos socioeconómicos, cruzando esta información en un segundo paso con toda la información disponible de las regiones de glaciares de Bolivia, el resultado de este proceso es una aproximación gruesa, pero sistemática, de la magnitud de los impactos socio-económicos para el país. Mediante este primer panorama de la problemática será posible indicar las necesidades de investigación futuras, tanto como señalizar elementos de una estrategia de respuesta o de mitigación.

Mientras que los efectos económicos permiten cálculos (por lo menos aproximados), el análisis de los efectos sociales necesariamente debe ser uno cualitativo en un primer momento.

Otro aspecto metodológico a mencionar es la dificultad de atribuir cualquier impacto social sobre la población al retroceso de los glaciares, tomando en cuenta las múltiples causas especialmente directas, del calentamiento global, y otros factores como por ejemplo la evolución de precios de productos agrícolas.

Sin embargo, se puede constatar que el impacto principal se da a través del recurso agua: menos caudal y estacionalidad del escurrimiento. Esto afecta al consumo humano, la agricultura, la biodiversidad, los ecosistemas, procesos productivos e industriales, la producción de energía hidroeléctrica e incluso hay el peligro de un empeoramiento de la calidad del agua.

La reducida disponibilidad del recurso agua conllevará, según las características locales, cambios en los patrones de asentamiento rural, un acelerado despoblamiento, un aumento de la migración hacia las ciudades, y cambios en el uso de la tierra.

\section{Resultados}

- Casi no existen investigaciones sobre impactos socio-económico del derretimiento de los glaciares, con la excepción de un primer estudio en curso, que estará indagando la relevancia del agua de origen glaciar para el suministro de agua potable para la demanda creciente de La PazEl Alto.

- El caso más notorio del retroceso glaciar para la población boliviana es el glaciar Chacaltaya, a unos pocos kilómetros de La Paz-El Alto. 
Cuando en los años 70 era lugar de competencias internacionales de esquí, hoy en día solo quedaron dos pequeños parches de hielo en la montaña.

- No hay prácticamente ninguna conciencia dentro de la población o el sistema político acerca de los problemas que el retroceso de los glaciares pudiera tener sobre las actividades humanas.

- En parte debido al hecho de que los glaciares bolivianos representan solamente una muy pequeña parte de los de América Latina, es muy probable que no se sentiría consecuencias de escala para los sistemas social o económico del país y de la región andina.

- Los impactos del retroceso de los glaciares muy probablemente serán de nivel local, en algunos casos afectando el espacio de varios municipios.

- Debido a los padrones de asentamiento humano y a una falta generalizada de infraestructura de valor económico en zonas de cuencas glaciares, parece haber relativamente poco riesgo de destrozos como consecuencia del derretimiento glaciar.

- Otro resultado es el impacto previsible sobre la diversidad biológica de lata montaña y la alteración de páramos y humedales como almacenes de agua y reguladores del clima local.

- De todo lo arriba mencionado, podemos deducir que el suministro de agua (potable) para la conglomeración urbana en crecimiento que es La Paz-El Alto, constituiría el tema más importante relativo al retroceso de los glaciares bolivianos.

\section{Discusión: Respuesta del sistema socio-político}

Tanto las entrevistas al azar, como un análisis de prensa y otros medios de comunicación muestran, que hasta la fecha prácticamente no hay ninguna conciencia pública acerca de los posibles impactos del derretimiento de los glaciares. En el nivel político-institucional la única excepción está dada por el Programa Nacional de Cambio Climático (PNCC) del Ministerio de Desarrollo Sostenible, que está financiando un primer estudio sobre el derretimiento de los glaciares y la disponibilidad de agua potable para los 1,5 millones de habitantes de la región metropolitana La Paz-El Alto.

El gobierno no tiene ninguna estrategia de mitigación u otros planes para enfrentar los efectos negativos del retroceso de los glaciares, inducidos por el cambio climático. Con mucha probabilidad el impacto del retroceso glaciar se sentiría más fuerte en el nivel local, lo que significa que las autoridades municipales tendrían que jugar un rol fundamental en este contexto. 


\section{Conclusiones: Necesidades futuras de investigación}

Los impactos socio-económicos se harán notar sobre todo en un nivel local, en algunos casos talvez incluso en escala regional, motivo por el cual se necesitarían estudios locales más detallados, en base a una delimitación de las áreas más proclives a sufrir impactos. Un elemento importante sería tanto el desarrollo de una metodología uniforme, cuanto una estimación global del porcentaje del escurrimiento glaciar comparado al escurrimiento total. Un segundo elemento sería poder contar con un análisis más detallado de los riesgos, correlacionando las cuencas glaciares principales con los padrones de asentamientos humanos y de distribución de infraestructura dentro de su alcance.

Aparte de la necesidad de investigaciones detalladas a niveles locales, se necesitarían mayores esfuerzos de investigación para pronosticar la disponibilidad futura de agua en las cuencas andinas a un nivel regional.

Considerando especialmente las tasas altas del retroceso de los glaciares pequeños, sería de gran utilidad poder contar con un inventario actualizado de los glaciares de Bolivia y la región de los Andes centrales, incluyendo datos actualizados del balance de masas, tomando en cuenta que los únicos datos disponibles a nivel de país datan de los anos 80 y a veces incluso de los 70 (JORDAN, 1991).

Para llevar adelante la discusión sobre los impactos tanto como las futuras acciones necesarias y pertinentes, es importante poder llegar a la identificación de elementos claves para la formulación de estrategias de mitigación y adaptación a nivel de cada país, que permitan minimizar los impactos del deshielo de los glaciares en especial sobre aquellas poblaciones más vulnerables (véase HOFFMANN, 2007).

\section{Agradecimientos}

Se agradece de manera especial a Bernard Francou de Great Ice / IRD y Edson Ramírez del Instituto de Hidraulica y Hidrología / UMSA y sus equipos de trabajo por el apoyo brindado.

\section{Información institucional}

El Instituto Boliviano de la Montaña es una fundación sin fines de lucro con sede en La Paz, cuya misión es contribuir al desarrollo sostenible de regiones de montaña en Bolivia a través de investigación científica, 
capacitación y asistencia técnica, intercambio de experiencias y la realización de proyectos. Más información: www.bolivian-mountains.org

\section{Referencias}

BRADLEY, R.S., et al (2006). Climate Change. Threats to Water Supplies in the Tropical Andes. Science 312: 1755-1756.

HOFFMANN D. (2005). Socio-economic impacts of glacier retreat in the Bolivian Andes. Exposición presentada en: Open Science Conference "Global Change in Mountain Regions". Perth, Scotland, U.K., 2-6 Octubre.

HOFFMANN D. (2007). Introducción sobre el impacto del retroceso de los glaciares y los recursos hídricos - presentación de las conclusiones de la conferencia regional de Quito. En: BMI (ed.): Memoria del foro-debate "Retroceso de los Glaciares y Recursos Hídricos en Bolivia - De la Investigación a la Acción". La Paz.

JORDAN E. (1991). Die Gletscher der bolivianischen Anden. Stuttgart: Franz Steiner. 365 p.

RAMÍREZ, E., FRANCOU, B., RIBSTEIN, P., DESCLOITRES, M., GUÉRIN, R., MENDOZA, J., GALLAIRE, R., POUYAUD, B. \& JORDAN, E. (2001). Small glaciers disappearing in the tropical Andes: a case study in Bolivia: Glaciar Chacaltaya (16 S). Journal of Glaciology. 47: 187-194.

WGMS (2007). http:/ / www.kms.dk/fags/ps09wgms.htm 